\title{
A Generalized Nonlinear Gronwall-Bellman Inequality with Maxima in Two Variables
}

\author{
Yong Yan \\ Department of Mathematics, Sichuan University for Nationalities, Kangding, Sichuan 626001, China
}

Correspondence should be addressed to Yong Yan; kdyan698@163.com

Received 15 November 2012; Accepted 20 January 2013

Academic Editor: Jitao Sun

Copyright (c) 2013 Yong Yan. This is an open access article distributed under the Creative Commons Attribution License, which permits unrestricted use, distribution, and reproduction in any medium, provided the original work is properly cited.

This paper deals with a generalized form of nonlinear retarded Gronwall-Bellman type integral inequality in which the maximum of the unknown function of two variables is involved. This form includes both a nonconstant term outside the integrals and more than one distinct nonlinear integrals. Requiring neither monotonicity nor separability of given functions, we apply a technique of monotonization to estimate the unknown function. Our result can be used to weaken conditions for some known results. We apply our result to a boundary value problem of a partial differential equation with maxima for uniqueness.

\section{Introduction}

The Gronwall-Bellman inequality $[1,2]$ plays an important role in the study of existence, uniqueness, boundedness, stability, invariant manifolds, and other qualitative properties of solutions of differential equations and integral equations. There can be found a lot of its generalizations in various cases from literatures (see, e.g., [3-18]). In 1956, Bihari [3] discussed the integral inequality

$$
u(t) \leq c+\int_{0}^{t} f(s) \omega(u(s)) d s, \quad t \geq 0
$$

where $c>0$ is a constant, $f$ is a continuous and nonnegative function, and $\omega$ is a continuous and nondecreasing positive function. Replacing $t$ by a function $b(t)$ in (1), Lipovan [4] investigated the retarded integral inequality

$$
\begin{aligned}
u(t) \leq & c+\int_{t_{0}}^{t} f(s) \omega(u(s)) d s \\
& +\int_{b\left(t_{0}\right)}^{b(t)} g(s) \omega(u(s)) d s, \quad t_{0} \leq t<t_{1} .
\end{aligned}
$$

Their results were further generalized by Agarwal et al. [5] to the inequality

$$
u(t) \leq a(t)+\sum_{i=1}^{n} \int_{b_{i}\left(t_{0}\right)}^{b_{i}(t)} f_{i}(t, s) \omega_{i}(u(s)) d s, \quad t_{0} \leq t<t_{1}
$$

where the constant $c$ is replaced with a function $a(t), b_{i}$ 's are continuously differentiable and nondecreasing functions, and $\omega_{i}$ 's are continuous and nondecreasing positive functions such that

$$
\omega_{1} \propto \omega_{2} \propto \cdots \propto \omega_{n}
$$

that is, each ratio $\omega_{i+1} / \omega_{i}$ is also nondecreasing on $A \subseteq \mathbb{R} \backslash\{0\}$, called in [6] that $\omega_{i+1}$ is stronger nondecreasing than $\omega_{i}$. On the basis of this work, Wang [7] considered the inequality of two variables

$$
\begin{aligned}
u^{p}(x, y) \leq & a(x, y) \\
& +\sum_{i=1}^{n} \int_{b_{i}\left(x_{0}\right)}^{b_{i}(x)} \int_{c_{i}\left(y_{0}\right)}^{c_{i}(y)} f_{i}(x, y, s, t) \omega_{i}(u(s, t)) d t d s,
\end{aligned}
$$

where the functions $a, f_{i}$, and $\omega_{i}$ are not required to be monotone, and those $\omega_{i}$ 's are not required to be stronger 
monotone than the one after the next as shown in (4). This inequality belongs to both the case of multivariables, to which great attentions [7-11] have been paid, and to the case that the left-hand side is a composition of the unknown function with a known function, in which Ou-Iang's idea [19] was applied [11-14]. He applied a technique of monotonization to construct a sequence of functions, made each function possess stronger monotonization than the previous one, and gave an estimate for the unknown function $u$.

On the other aspect, many problems in the control theory can be modeled in the form of differential equations with the maxima of the unknown function [20-22]. In connection with the development of the theory of differential equations with maxima (see, e.g., $[20,21,23]$ ) and partial differential equations with maxima $[24,25]$, a new type of integral inequalities with maxima is required, respectively. There have been given some results for integral inequalities containing the maxima of the unknown function [23, 26-28]. Concretely, in 2012, Bohner et al. [26] discussed the following system of integral inequalities:

$$
\begin{gathered}
\varphi(u(t)) \leq a(t)+\sum_{i=1}^{m} \int_{\alpha_{i}\left(t_{0}\right)}^{\alpha_{i}(t)} f_{i}(s) u^{p}(s) \omega_{i}(u(s)) d s \\
+\sum_{j=m+1}^{m+n} \int_{\alpha_{j}\left(t_{0}\right)}^{\alpha_{j}(t)} f_{j}(s) u^{p}(s) \\
\times \omega_{j}\left(\max _{\xi \in[s-h, s]} g(u(\xi))\right) d s \\
u(t) \leq \psi(t),
\end{gathered}
$$

where $a, f_{i}^{\prime}$ 's, $\omega_{i}$ 's, $\varphi$, and $\psi$ are nonnegative continuous functions and $\alpha_{i}$ 's are nonnegative continuously differentiable and nondecreasing functions. They required that $a(t) \geq 1$, $\varphi$ is $C^{1}$ on $\mathbb{R}_{+}:=[0,+\infty)$ and increasing such that $\varphi(t x) \geq$ $t \varphi(x)$ for $0 \leq t \leq 1$, and $\omega_{i}$ satisfies the following: (i) $\omega_{i} \in$ $C^{1}\left(\mathbb{R}_{+}, \mathbb{R}_{+}\right)$is an increasing function, and (ii) $\omega_{i}(t x) \geq t \omega_{i}(x)$ for all $0 \leq t \leq 1$ and $x>0$. Bainov and Hristova [23] considered the following system:

$$
\begin{gathered}
u(x, y) \leq a(x, y)+\int_{x_{0}}^{x} \int_{y_{0}}^{y} f(s, t) u^{p}(s, t) d t d s \\
+\int_{\alpha\left(x_{0}\right)}^{\alpha(x)} \int_{y_{0}}^{y} h(s, t)\left(\max _{\xi \in[s-h, s]} u^{p}(\xi, t)\right) d t d s \\
u(x, y) \leq \psi(x, y)
\end{gathered}
$$

where $a(x, y)$ is nonnegative and nondecreasing in both of its arguments, $f, h$, and $\psi$ are continuous and nonnegative functions, and $p \in(0,1]$.
In this paper, we consider the system of integral inequalities as follows:

$$
\begin{aligned}
& \varphi(u(x, y)) \\
& \leq a(x, y) \\
& +\sum_{i=1}^{n} \int_{\alpha_{i}\left(x_{0}\right)}^{\alpha_{i}(x)} \int_{\beta_{i}\left(y_{0}\right)}^{\beta_{i}(y)} f_{i}(x, y, s, t) \omega_{i}(u(s, t)) d t d s \\
& +\sum_{j=n+1}^{n+m} \int_{\alpha_{j}\left(x_{0}\right)}^{\alpha_{j}(x)} \int_{\beta_{j}\left(y_{0}\right)}^{\beta_{j}(y)} f_{j}(x, y, s, t) \\
& \quad \times \omega_{j}\left(\max _{\xi \in[s-h, s]} g(u(\xi, t))\right) d t d s, \\
& \quad(x, y) \in\left[x_{0}, x_{1}\right) \times\left[y_{0}, y_{1}\right), \\
& u(x, y) \leq \psi(x, y), \quad(x, y) \in\left[\alpha_{*}\left(x_{0}\right)-h, x_{0}\right] \times\left[y_{0}, y_{1}\right),
\end{aligned}
$$

where $a, f_{i}$ 's, $\omega_{i}$ 's, and $g$ are continuous and nonnegative functions, $\alpha_{i}$ 's and $\beta_{i}$ 's are nonnegative continuously differentiable and nondecreasing functions, and $\alpha_{*}\left(x_{0}\right)$ := $\min _{1 \leq i \leq m+n} \alpha_{i}\left(x_{0}\right)$. As required in previous works [27-29], we suppose that $0 \leq \alpha_{i}(t) \leq t, 0 \leq \beta_{i}(t) \leq t, h>0$ is constant. In this paper, we require neither monotonicity of $a, \omega_{i}$ 's, $f_{i}$ 's, and $g$ nor $a(x, y) \geq 1$. We monotonize those $\omega_{i}$ 's to make a sequence of functions in which each one possesses stronger monotonicity than the previous one so as to give an estimation for the unknown function. We can use our result to discuss inequalities (6) and (7), giving the stronger results under weaker conditions. We finally apply the obtained result to a boundary value problem of a partial differential equation with maxima for uniqueness.

\section{Main Result}

Consider system (8) of integral inequalities with $x_{0}<x_{1}$ and $y_{0}<y_{1}$ in $\mathbb{R}_{+}:=[0, \infty)$. Let $\Lambda:=\left[x_{0}, x_{1}\right) \times\left[y_{0}, y_{1}\right), \Omega:=$ $\left[\alpha_{*}\left(x_{0}\right)-h, x_{0}\right] \times\left[y_{0}, y_{1}\right)$. Suppose that

$\left(\mathrm{H}_{1}\right) \alpha_{i}:\left[x_{0}, x_{1}\right) \rightarrow \mathbb{R}_{+}(i=1,2, \ldots, m+n)$ and $\beta_{i}:\left[y_{0}, y_{1}\right) \rightarrow\left[y_{0}, y_{1}\right), i=1,2, \ldots, m+$ $n$, are nondecreasing such that $\alpha_{i}(x) \leq x$ on $\left[x_{0}, x_{1}\right), \beta_{i}(y) \leq y$ on $\left[y_{0}, y_{1}\right)$ and $\beta_{i}\left(y_{0}\right)=y_{0}$;

$\left(\mathrm{H}_{2}\right)$ all $f_{i}^{\prime}$ s $(i=1,2, \ldots, m+n)$ are continuous and nonnegative functions on $\Lambda \times\left[\alpha_{*}\left(x_{0}\right), x_{1}\right) \times\left[y_{0}, y_{1}\right)$;

$\left(\mathrm{H}_{3}\right) g, \varphi: \mathbb{R}_{+} \rightarrow \mathbb{R}_{+}$and $\psi:\left[\alpha_{*}\left(x_{0}\right)-h, x_{1}\right) \rightarrow \mathbb{R}_{+}$ are continuous, and $\varphi$ is strictly increasing such that $\lim _{t \rightarrow \infty} \varphi(t)=+\infty$;

$\left(\mathrm{H}_{4}\right)$ all $\omega_{i}$ 's $(i=1,2, \ldots, m+n)$ are continuous on $\mathbb{R}_{+}$and positive on $(0,+\infty)$;

$\left(\mathrm{H}_{5}\right) a(x, y)$ is a continuous and nonnegative function on $\Lambda$. 
For those $\omega_{i}$ 's given in $\left(\mathrm{H}_{4}\right)$, define $\widetilde{\omega}_{i}(t), i=1, \ldots, m+n$, inductively by

$$
\begin{gathered}
\widetilde{\omega}_{1}(t):=\max _{\tau \in[0, t]}\left\{\omega_{1}(\tau)\right\}, \\
\widetilde{\omega}_{i+1}(t):=\max _{\tau \in[0, t]}\left\{\frac{\omega_{i+1}(\tau)}{\widetilde{\omega}_{i}(\tau)+\epsilon_{i}}\right\} \widetilde{\omega}_{i}(t),
\end{gathered}
$$

for $i=1,2, \ldots, m-1$ and

$$
\begin{gathered}
\widetilde{\omega}_{m+1}(t):=\max _{\tau \in[0, t]}\left\{\frac{\widehat{\omega}_{m+1}\left(\max _{s \in[0, \tau]}\{g(s)\}\right)}{\widetilde{\omega}_{m}(\tau)+\epsilon_{m}}\right\} \widetilde{\omega}_{m}(t), \\
\widetilde{\omega}_{j+1}(t):=\max _{\tau \in[0, t]}\left\{\frac{\widehat{\omega}_{j+1}\left(\max _{s \in[0, \tau]}\{g(s)\}\right)}{\widetilde{\omega}_{j}(\tau)+\epsilon_{j}}\right\} \widetilde{\omega}_{j}(t),
\end{gathered}
$$

for $j=m+1, \ldots, m+n$, where $\widehat{\omega}_{j}(t):=\max _{\tau \in[0, t]}\left\{\omega_{j}(\tau)\right\}$ for $j=m+1, \ldots, m+n, \epsilon_{i}:=\varepsilon_{1}$ if $\widetilde{\omega}_{i}(0)=0$ or $:=0$ if $\widetilde{\omega}_{i}(0) \neq 0$ for $i=1,2, \ldots, m+n-1$, and $\varepsilon_{1}>0$ be a given very small constant.

Theorem 1. Suppose that $\left(H_{1}\right)-\left(H_{5}\right)$ hold, $\max _{s \in\left[\alpha_{*}\left(x_{0}\right)-h, x_{0}\right]} \psi(s, y) \leq \varphi^{-1}\left(a\left(x_{0}, y\right)\right)$ for all $y \in\left[y_{0}, y_{1}\right)$ and $u \in C\left(\Omega, \mathbb{R}_{+}\right)$satisfies the system (8) of integral inequalities. Then,

$$
u(x, y) \leq \varphi^{-1}\left(W_{m+n}^{-1}\left(\Omega_{m+n}(x, y)\right)\right)
$$

for all $(x, y) \in\left[x_{0}, X_{1}\right) \times\left[y_{0}, Y_{1}\right)$, where

$$
\begin{aligned}
\Omega_{i}(x, y):= & W_{i}\left(r_{i}(x, y)\right) \\
& +\int_{\alpha_{i}\left(x_{0}\right)}^{\alpha_{i}(x)} \int_{\beta_{i}\left(y_{0}\right)}^{\beta_{i}(y)} \max _{(l, \xi) \in\left[x_{0}, x\right] \times\left[y_{0}, y\right]} f_{i}(l, \xi, s, t) d t d s,
\end{aligned}
$$

$W_{i}^{-1}$ is the inverse of the function

$$
W_{i}(u):=\int_{u_{i}}^{u} \frac{d s}{\widetilde{\omega}_{i}\left(\varphi^{-1}(s)\right)}, \quad u \geq u_{i}, i=1,2, \ldots, m+n
$$

$u_{i}>0$ is a given constant, $\widetilde{\omega}_{i}$ is defined just before the theorem, and $r_{i}(x, y)$ is defined recursively by

$$
\begin{gathered}
r_{1}(x, y)=\max _{(\iota, \xi) \in\left[x_{0}, x\right] \times\left[y_{0}, y\right]} a(\iota, \xi), \\
r_{i+1}(x, y)=W_{i}^{-1}\left(\Omega_{i}(x, y)\right),
\end{gathered}
$$

for $i=1,2, \ldots, m+n-1$, and $X_{1} \in\left[x_{0}, x_{1}\right), Y_{1} \in\left[y_{0}, y_{1}\right)$ are chosen such that

$$
\Omega_{i}\left(X_{1}, Y_{1}\right) \leq \int_{u_{i}}^{\infty} \frac{d s}{\widetilde{\omega}_{i}\left(\varphi^{-1}(s)\right)},
$$

for $i=1,2, \ldots, m+n$.

For the special choice that $n=m=1, \omega_{1}(s)=s^{p}, \omega_{2}(s)=$ $s, f_{1}(x, y, s, t)=f(s, t), f_{2}(x, y, s, t)=h(s, t), \alpha_{1}(s)=s$, $\alpha_{2}(s)=\alpha(s), g(s)=s^{p}$, and $\beta_{1}(s)=\beta_{2}(s)=s$, where $\alpha$ is a nonnegative continuously differentiable and nondecreasing function, Theorem 1 gives an estimate for the unknown $u$ in the system (7). we require neither the monotonicity of $a$ nor the monotonicity of $\omega_{i}$. Obviously, Lemma 2 and Theorem 1 are applicable to more general forms than Corollary 2.3.4 in [23]. Even if $\omega_{i}(s)$ is enlarged to $\max _{1<i<m+n} \omega_{i}(s)$ such that (8) is changed into the form of (2.1) in [29], where $m=n=1$, our theorem gives a better estimate. For example, the system of inequalities

$$
\begin{aligned}
& u(x, y) \leq 3+4 \int_{0}^{x} \int_{0}^{y} t s \sqrt{u(s, t)+1} d t d s \\
& +4 \int_{0}^{\sqrt{x}} \int_{0}^{y} t s\left(\max _{\xi \in[s-h, s]} u(\xi, t)+1\right) d t d s, \\
& \quad(x, y) \in\left[0, x_{1}\right) \times\left[0, y_{1}\right), \\
& u(x, y) \leq x+3, \quad(x, y) \in[-h, 0] \times\left[0, y_{1}\right),
\end{aligned}
$$

implies that

$$
\begin{aligned}
& u(x, y) \leq 3+4 \int_{0}^{x} \int_{0}^{y} t s(u(s, t)+1) d t d s \\
& +4 \int_{0}^{\sqrt{x}} \int_{0}^{y} t s\left(\max _{\xi \in[s-h, s]} u(\xi, t)+1\right) d t d s, \\
& \quad(x, y) \in\left[0, x_{1}\right) \times\left[0, y_{1}\right), \\
& u(x, y) \leq x+3, \quad(x, y) \in[-h, 0] \times\left[0, y_{1}\right),
\end{aligned}
$$

by enlarging $\sqrt{s+1}$ to $s+1$. Applying Theorem 1 , we obtain

$$
u(x, y) \leq \frac{\left(x^{2} y^{2}+4\right)^{2}}{4} e^{x y^{2}}, \quad(x, y) \in\left[0, x_{1}\right) \times\left[0, y_{1}\right)
$$


On the other hand, Theorem 2.2 of [29] gives from (17) that

$$
u(t) \leq 4 e^{\left(x^{2} y^{2}+x y^{2}\right)}, \quad(x, y) \in\left[0, x_{1}\right) \times\left[0, y_{1}\right)
$$

Clearly, (18) is sharper than (19) for large $x$ and $y$. lemma.

In order to prove Theorem 1, we need the following

\section{Lemma 2. Suppose that}

(C1) $\alpha_{i}:\left[x_{0}, x_{1}\right) \rightarrow \mathbb{R}_{+}(i=1,2, \ldots, m+n)$ and $\beta_{i}:$ $\left[y_{0}, y_{1}\right) \rightarrow \mathbb{R}_{+}(i=1,2, \ldots, m+n)$ are nondecreasing such that $\alpha_{i}(x) \leq x$ on $\left[\alpha_{*}\left(x_{0}\right), x_{1}\right)$ and $\beta_{i}(y) \leq y$ on $\left[y_{0}, y_{1}\right)$ and $\beta_{i}\left(y_{0}\right)=y_{0}$;

(C2) $\psi \in C\left(\left[\alpha_{*}\left(x_{0}\right)-h, x_{1}\right) \times\left[y_{0}, y_{1}\right), \mathbb{R}_{+}\right), b_{i} \in C\left(\left[\alpha_{*}\left(x_{0}\right)\right.\right.$, $\left.\left.x_{1}\right) \times\left[y_{0}, y_{1}\right), \mathbb{R}_{+}\right)$for $i=1,2, \ldots, m+n ;$

(C3) all $h_{i}$ 's $(i=1,2, \ldots, m+n)$ are continuous and nondecreasing on $\mathbb{R}_{+}$and positive on $(0,+\infty)$ such that $h_{1} \propto h_{2} \propto \ldots \propto h_{m+n}$;

(C4) $a(x, y)$ is continuously differentiable in $x$ and $y$, nonnegative on $\left[\alpha_{*}\left(x_{0}\right), x_{1}\right) \times\left[y_{0}, y_{1}\right)$, and $\max _{s \in\left[\alpha_{*}\left(x_{0}\right)-h, x_{1}\right]} \psi(s, y) \leq a\left(x_{0}, y\right)$ for all $y \in\left[y_{0}, y_{1}\right)$.

If $u \in C\left(\left[\alpha_{*}\left(x_{0}\right)-h, x_{1}\right) \times\left[y_{0}, y_{1}\right), \mathbb{R}_{+}\right)$satisfies the system of inequalities as follows:

$$
\begin{gathered}
u(x, y) \\
\leq a(x, y)+\sum_{i=1}^{m} \int_{\alpha_{i}\left(x_{0}\right)}^{\alpha_{i}(x)} \int_{\beta_{i}\left(y_{0}\right)}^{\beta_{i}(y)} b_{i}(s, t) h_{i}(u(s, t)) d t d s \\
+\sum_{j=m+1}^{m+n} \int_{\alpha_{j}\left(x_{0}\right)}^{\alpha_{j}(x)} \int_{\beta_{j}\left(y_{0}\right)}^{\beta_{j}(y)} h_{j}(s, t) h_{j}\left(\max _{\xi \in[s-h, s]} u(\xi, t)\right) d t d s, \quad(x, y) \in \Lambda, \\
u(x, y) \leq \psi(x, y), \quad(x, y) \in \Omega,
\end{gathered}
$$

then

$$
\begin{aligned}
u(x, y) \leq H_{m+n}^{-1}( & H_{m+n}\left(\gamma_{m+n}(x, y)\right) \\
& \left.+\int_{\alpha_{m+n}\left(x_{0}\right)}^{\alpha_{m+n}(x)} \int_{\beta_{m+n}\left(y_{0}\right)}^{\beta_{m+n}(y)} h_{m+n}(s, t) d t d s\right),
\end{aligned}
$$

for all $(x, y) \in\left[x_{0}, X^{*}\right) \times\left[y_{0}, Y^{*}\right)$, where $H_{i}^{-1}$ is the inverse of the function

$$
H_{i}(u):=\int_{u_{i}}^{u} \frac{d x}{\omega_{i}(x)}, \quad u \geq u_{i}, i=1,2, \ldots, m+n,
$$

$u_{i}>0$ is a given constant, and $\gamma_{i}(x, y)$ is defined recursively by

$$
\begin{aligned}
\gamma_{1}(x, y):=a\left(x_{0}, y_{0}\right)+\int_{x_{0}}^{x}\left|a_{x}(t, y)\right| d t+\int_{y_{0}}^{y}\left|a_{y}(x, s)\right| d s, \\
\gamma_{i+1}(x, y):=H_{i}^{-1}\left(H_{i}\left(\gamma_{i}(x, y)\right)+\int_{\alpha_{i}\left(t_{0}\right)}^{\alpha_{i}(t)} \int_{\beta_{i}\left(t_{0}\right)}^{\beta_{i}(t)} b_{i}(s, t) d t d s\right)
\end{aligned}
$$

for $i=1,2, \ldots, m+n-1$, and $x_{0} \leq X^{*}<x_{1}, y_{0} \leq Y^{*}<y_{1}$ are chosen such that

$$
H_{i}\left(\gamma_{i}\left(X^{*}, Y^{*}\right)\right)+\int_{\alpha_{i}\left(x_{0}\right)}^{\alpha_{i}\left(X^{*}\right)} \int_{\beta_{i}\left(y_{0}\right)}^{\beta_{i}\left(Y^{*}\right)} b_{i}(s, t) d t d s \leq \int_{u_{i}}^{\infty} \frac{d s}{h_{i}(s)},
$$

for $i=1,2, \ldots, m+n$.

Proof. From (23), we see that $\gamma_{1}(x, y)$ is nondecreasing on $\Lambda$, $a(x, y) \leq \gamma_{1}(x, y)$, and $\max _{s \in\left[\alpha_{*}\left(x_{0}\right)-h, x_{1}\right]} \psi(s, y) \leq a\left(x_{0}, y\right) \leq$ $\gamma_{1}\left(x_{0}, y\right)$ for $y \in\left[y_{0}, y_{1}\right)$. It implies from (20) that

$$
\begin{gathered}
u(x, y) \leq \gamma_{1}(x, y)+\sum_{i=1}^{m} \int_{\alpha_{i}\left(x_{0}\right)}^{\alpha_{i}(x)} \int_{\beta_{i}\left(y_{0}\right)}^{\beta_{i}(y)} b_{i}(s, t) h_{i}(u(s, t)) d t d s \\
+\sum_{j=m+1}^{m+n} \int_{\alpha_{j}\left(x_{0}\right)}^{\alpha_{j}(x)} \int_{\beta_{j}\left(y_{0}\right)}^{\beta_{j}(y)} b_{j}(s, t) \\
\times h_{j}\left(\max _{\xi \in[s-h, s]} u(\xi, t)\right) d t d s
\end{gathered}
$$

(21) for all $(x, y) \in \Lambda$. Let

$$
z(x, y):= \begin{cases}\gamma_{1}(x, y)+\sum_{i=1}^{m} \int_{\alpha_{i}\left(x_{0}\right)}^{\alpha_{i}(x)} \int_{\beta_{i}\left(y_{0}\right)}^{\beta_{i}(y)} b_{i}(s, t) h_{i}(u(s, t)) d t d s & \\ +\sum_{j=m+1}^{m+n} \int_{\alpha_{j}\left(x_{0}\right)}^{\alpha_{j}(x)} \int_{\beta_{i}\left(y_{0}\right)}^{\beta_{i}(y)} b_{j}(s, t) h_{j}\left(\max _{\xi \in[s-h, s]} u(\xi, t)\right) d t d s, \quad(x, y) \in \Lambda, \\ \gamma_{1}\left(x_{0}, y\right), & (x, y) \in \Omega .\end{cases}
$$


Clearly, $z(x, y)$ is nondecreasing in $x$. Then, we have

$$
\begin{gathered}
u(x, y) \leq z(x, y), \quad(x, y) \in\left[\alpha_{*}\left(x_{0}\right)-h, \xi\right) \times\left[y_{0}, \eta\right) \\
\max _{\xi \in[s-h, s]} u(\xi, y) \leq \max _{\xi \in[s-h, s]} z(\xi, y)=z(s, y) \\
(s, y) \in\left[\alpha_{*}\left(x_{0}\right), x_{1}\right) \times\left[y_{0}, y_{1}\right) .
\end{gathered}
$$

From (25), (27), and (28) and the definition of $z(x, y)$ on $\Lambda$, we get

$$
\begin{aligned}
z(x, y) \leq & \gamma_{1}(x, y)+\sum_{i=1}^{m} \int_{\alpha_{i}\left(x_{0}\right)}^{\alpha_{i}(x)} \int_{\beta_{i}\left(y_{0}\right)}^{\beta_{i}(y)} b_{i}(s, t) h_{i}(u(s, t)) d t d s \\
& +\sum_{j=m+1}^{m+n} \int_{\alpha_{j}\left(x_{0}\right)}^{\alpha_{j}(x)} \int_{\beta_{i}\left(y_{0}\right)}^{\beta_{i}(y)} b_{j}(s, t) h_{j}(z(s, t)) d t d s .
\end{aligned}
$$

Applying Theorem 1 of [7] to the case that $f_{i}(x, y, s, t)=$ $b_{i}(s, t), a(x, y)=\gamma_{1}(x, y), p=1$, and $\omega_{i}(t)=h_{i}(t), i=$ $1,2, \ldots, m+n$, we obtain (21) from (28). This completes the proof.

Proof of Theorem 1. First of all, we monotonize some given functions $f_{i}, \omega_{i}, g$, and $a$ in the system (8) of integral inequalities. Let

$$
\begin{gathered}
\tilde{g}(t):=\max _{\tau \in[0, t]}\{g(\tau)\}, \quad t \geq 0, \\
\widetilde{a}(x, y):=\max _{(\tau, \xi) \in\left[x_{0}, x\right] \times\left[y_{0}, y\right]}\{a(\tau, \xi)\}, \\
(x, y) \in\left[x_{0}, x_{1}\right) \times\left[y_{0}, y_{1}\right) .
\end{gathered}
$$

From (13), we see that the function $W_{i}$ is strictly increasing, and therefore its inverse $W_{i}^{-1}$ is well defined, continuous, and increasing in its domain. The sequence $\left\{\widetilde{\omega}_{i}(t)\right\}$, defined by $\omega_{i}(s)$, consists of nondecreasing nonnegative functions on $\mathbb{R}_{+}$ and satisfies

$$
\begin{gathered}
\omega_{i}(t) \leq \widetilde{\omega}_{i}(t), \quad i=1,2, \ldots, m, \\
\omega_{i}(t) \leq \widehat{\omega}_{i}(t), \quad i=m+1, \ldots, m+n, \\
\widehat{\omega}_{i}(\widetilde{g}(t)) \leq \widetilde{\omega}_{i}(t), \quad i=m+1, \ldots, m+n .
\end{gathered}
$$

Moreover,

$$
\widetilde{\omega}_{i} \propto \widetilde{\omega}_{i+1}, \quad i=1,2, \ldots, m+n,
$$

because the ratios $\widetilde{\omega}_{i+1} / \widetilde{\omega}_{i}, i=1,2, \ldots, m+n$, are all nondecreasing. Furthermore, let

$$
\tilde{f}_{i}(x, y, s, t):=\max _{(\iota, \xi) \in\left[x_{0}, x\right] \times\left[y_{0}, y\right]} f_{i}(\iota, \xi, s, t),
$$

which is nondecreasing in $x$ and $y$ for each fixed $t$ and $s$ and satisfies $\widetilde{f}_{i}(x, y, s, t) \geq f_{i}(x, y, s, t) \geq 0$ for all $i=1,2, \ldots, m+$ $n$. The monotonicity of $\widetilde{g}$ implies that

$$
\max _{\xi \in[s-h, s]} g(u(\xi, y)) \leq \max _{\xi \in[s-h, s]} \tilde{g}(u(\xi, y)) \leq \widetilde{g}\left(\max _{\xi \in[s-h, s]} u(\xi, y)\right)
$$

for $(s, y) \in\left[\alpha_{*}\left(x_{0}\right)-h, x_{1}\right) \times\left[y_{0}, y_{1}\right)$. From (8) and the definition of $\tilde{f}_{i}(x, y, s, t)$, we obtain

$$
\begin{aligned}
\varphi(u(x, y)) \leq & \widetilde{a}(x, y) \\
& +\sum_{i=1}^{n} \int_{\alpha_{i}\left(x_{0}\right)}^{\alpha_{i}(x)} \int_{\beta_{i}\left(y_{0}\right)}^{\beta_{i}(y)} \tilde{f}_{i}(x, y, s, t) \\
& \times \omega_{i}(u(s, t)) d t d s \\
& +\sum_{j=n+1}^{n+m} \int_{\alpha_{j}\left(x_{0}\right)}^{\alpha_{j}(x)} \int_{\beta_{j}\left(y_{0}\right)}^{\beta_{j}(y)} \tilde{f}_{j}(x, y, s, t) \\
& \times \omega_{j}\left(\max _{\xi \in[s-h, s]} g(u(\xi, t))\right) d t d s, \quad(x, y) \in \Lambda, \\
& u(x, y) \leq \psi(x, y), \quad(x, y) \in \Omega .
\end{aligned}
$$

Concerning (34), we consider the auxiliary system of inequalities

$$
\begin{aligned}
& \varphi(u(x, y)) \leq \tilde{a}(X, Y) \\
&+\sum_{i=1}^{n} \int_{\alpha_{i}\left(x_{0}\right)}^{\alpha_{i}(x)} \int_{\beta_{i}\left(y_{0}\right)}^{\beta_{i}(y)} \tilde{f}_{i}(X, Y, s, t) \\
& \times \omega_{i}(u(s, t)) d t d s \\
&+ \sum_{j=1}^{m} \int_{\alpha_{j}\left(x_{0}\right)}^{\alpha_{j}(x)} \int_{\beta_{i}\left(y_{0}\right)}^{\beta_{i}(y)} \tilde{f}_{j}(X, Y, s, t) \\
& \times \omega_{j}\left(\max _{\xi \in[s-h, s]} g(u(\xi, t))\right) d t d s, \\
&(x, y) \in\left[x_{0}, X\right) \times\left[y_{0}, Y\right), \\
& u(x, y) \leq \psi(x, y), \quad(x, y) \in \Omega,
\end{aligned}
$$

where $x_{0} \leq X \leq X_{1}$ and $y_{0} \leq Y \leq Y_{1}$ are chosen arbitrarily, and claim

$$
u(x, y) \leq \varphi^{-1}\left(W_{m+n}^{-1}\left(\widetilde{\Omega}_{i}(X, Y, x, y)\right)\right),
$$

for all $x_{0} \leq x \leq \min \left\{X, X_{2}\right\}, y_{0} \leq y \leq \min \left\{Y, Y_{2}\right\}$, where

$$
\widetilde{\Omega}_{i}(X, Y, x, y):=W_{i}\left(\widetilde{r}_{i}(X, Y, x, y)\right)
$$

$$
+\int_{\alpha_{i}\left(x_{0}\right)}^{\alpha_{i}(x)} \int_{\beta_{i}\left(y_{0}\right)}^{\beta_{i}(y)} \tilde{f}(X, Y, s, t) d t d s
$$

$i=1,2, \ldots, m+n, \widetilde{r}_{i}(X, Y, x, y)$ is defined inductively by

$$
\begin{gathered}
\widetilde{r}_{1}(X, Y, x, y):=\widetilde{a}(X, Y), \\
\widetilde{r}_{i+1}(X, Y, x, y):=W_{i}^{-1}\left(W_{i}\left(\widetilde{\Omega}_{i}(X, Y, x, y)\right)\right),
\end{gathered}
$$


for $i=1,2, \ldots, m+n-1$, and $X_{2}, Y_{2}$ are chosen such that

$$
\widetilde{\Omega}_{i}\left(X, Y, X_{2}, Y_{2}\right) \leq \int_{u_{i}}^{\infty} \frac{d s}{\widetilde{\omega}_{i}\left(\varphi^{-1}(s)\right)},
$$

for $i=1,2, \ldots, m+n$.

Notice that we may take $X_{2}=X_{1}$ and $Y_{2}=Y_{1}$. In fact, the monotonicity that $\widetilde{r}_{i}(X, Y, x, y)$ and $\tilde{f}_{i}(X, Y, x, y)$ are both nondecreasing in $X$ and $Y$ for fixed $x, y$. Furthermore, it is easy to check that $\widetilde{r}_{i+1}(X, Y, X, Y)=r_{i}(X, Y)$, for $i=$ $1,2, \ldots, m+n$. If $X_{2}, Y_{2}$ are replaced with $X_{1}, Y_{1}$, respectively, on the left side of (39), we get from (15) that

$$
\begin{aligned}
\widetilde{\Omega}_{i}\left(X, Y, X_{1}, Y_{1}\right)= & W_{i}\left(\widetilde{r}_{i}\left(X, Y, X_{1}, Y_{1}\right)\right) \\
& +\int_{\alpha_{i}\left(x_{0}\right)}^{\alpha_{i}\left(X_{1}\right)} \int_{\beta_{i}\left(y_{0}\right)}^{\beta_{i}\left(Y_{1}\right)} \tilde{f}(X, Y, s, t) d t d s \\
\leq & W_{i}\left(\widetilde{r}_{i}\left(X_{1}, Y_{1}, X_{1}, Y_{1}\right)\right) \\
& +\int_{\alpha_{i}\left(x_{0}\right)}^{\alpha_{i}\left(X_{1}\right)} \int_{\beta_{i}\left(y_{0}\right)}^{\beta_{i}\left(Y_{1}\right)} \tilde{f}\left(X_{1}, Y_{1}, s, t\right) d t d s \\
= & r_{i}\left(X_{1}, Y_{1}\right) \\
& +\int_{\alpha_{i}\left(x_{0}\right)}^{\alpha_{i}\left(X_{1}\right)} \int_{\beta_{i}\left(y_{0}\right)}^{\beta_{i}\left(Y_{1}\right)} \tilde{f}\left(X_{1}, Y_{1}, s, t\right) d t d s \\
= & \Omega_{i}\left(X_{1}, Y_{1}\right) \\
\leq & \int_{u_{i}}^{\infty} \frac{d s}{\widetilde{\omega}_{i}\left(\varphi^{-1}(s)\right)} .
\end{aligned}
$$

Thus, it means that we can take $X_{2}=X_{1}, Y_{2}=Y_{1}$.
Now, we prove (36) by induction. From (33), (35), and the definitions of $\widetilde{g}(t), \widetilde{\omega}_{i}(t)$, and $\widehat{\omega}_{i}(t)$, we obtain

$$
\begin{aligned}
\varphi(u & (x, y)) \\
\leq & \tilde{a}(X, Y) \\
& +\sum_{i=1}^{m} \int_{\alpha_{i}\left(x_{0}\right)}^{\alpha_{i}(x)} \int_{y_{0}}^{y} \widetilde{f}_{i}(X, Y, s, t) \omega_{i}(u(s, t)) \\
& +\sum_{j=m+1}^{m+n} \int_{\alpha_{j}\left(x_{0}\right)}^{\alpha_{j}(x)} \int_{y_{0}}^{y} \widetilde{f}_{j}(X, Y, s, t) \\
\leq & \widetilde{a}(X, Y) \\
& +\sum_{i=1}^{m} \int_{\alpha_{i}\left(x_{0}\right)}^{\alpha_{i}(x)} \int_{\beta_{i}\left(y_{0}\right)}^{\beta_{i}(y)} \widetilde{f}_{i}(X, Y, s, t) \widetilde{\omega}_{i}(u(s)) d s \\
& +\sum_{j=m+1}^{m+n} \int_{\alpha_{j}\left(x_{0}\right)}^{\alpha_{j}(x)} \int_{\beta_{j}\left(y_{0}\right)}^{\beta_{j}(y)} \tilde{f}_{j}(X, Y, s, t) \\
& +\widetilde{\omega}_{j}\left(\max _{\xi \in[s-h, s]} u(\xi)\right) d t d s, t s \\
&
\end{aligned}
$$

for all $(x, y) \in\left[x_{0}, X\right) \times\left[y_{0}, Y\right)$, where $x_{0} \leq X \leq X_{1}$ and $y_{0} \leq$ $Y \leq Y_{1}$ are chosen arbitrarily. Since $\max _{s \in\left[\alpha_{*}\left(x_{0}\right)-h, x_{0}\right]} \psi(s, y) \leq$ $\varphi^{-1}\left(a\left(x_{0}, y\right)\right)$ and $a\left(x_{0}, y\right) \leq \widetilde{a}\left(x_{0}, y\right) \leq \widetilde{a}(X, Y)$, we have $\max _{s \in\left[\alpha_{*}\left(x_{0}\right)-h, x_{0}\right]} \psi(s, y) \leq \varphi^{-1}(\widetilde{a}(X, Y))$. Define a function $z(x, y):\left[\alpha_{*}\left(x_{0}\right)-h, X\right) \times\left[y_{0}, Y\right) \rightarrow \mathbb{R}_{+}$by

$$
\begin{aligned}
& z(x, y) \\
& = \begin{cases}\widetilde{a}(X, Y)+\sum_{i=1}^{m} \int_{\alpha_{i}\left(x_{0}\right)}^{\alpha_{i}(x)} \int_{\beta_{i}\left(y_{0}\right)}^{\beta_{i}(y)} \widetilde{f}_{i}(X, Y, s, t) \widetilde{\omega}_{i}(u(s, t)) d t d s \\
\quad+\sum_{j=m+1}^{m+n} \int_{\alpha_{j}\left(x_{0}\right)}^{\alpha_{j}(x)} \int_{\beta_{i}\left(y_{0}\right)}^{\beta_{i}(y)} \tilde{f}_{j}(s, t) \widetilde{\omega}_{j}\left(\max _{\xi \in[s-h, s]} u(\xi, t)\right) d t d s, & (x, y) \in\left[x_{0}, X\right) \times\left[y_{0}, Y\right), \\
\tilde{a}(X, Y), & (x, y) \in\left[\alpha_{*}\left(x_{0}\right)-h, x_{0}\right] \times\left[y_{0}, Y\right) .\end{cases}
\end{aligned}
$$

Clearly, $z(x, y)$ is nondecreasing in $x$. By (41) and the definition of $z(x, y)$, we have

$$
\begin{aligned}
& u(x, y) \leq \varphi^{-1}(z(x, y)), \\
& \quad(x, y) \in\left[\alpha_{*}\left(x_{0}\right)-h, X\right) \times\left[y_{0}, Y\right) .
\end{aligned}
$$

Then noting that $z(x, y)$ is nondecreasing and $\varphi(t)$ is strictly increasing, from (43), we obtain

$$
\begin{aligned}
\max _{\xi \in[s-h, s]} u(\xi, y) & \leq \max _{\xi \in[s-h, s]} \varphi^{-1}(z(\xi, y)) \leq \max _{\xi \in[s-h, s]} \varphi^{-1}(z(s, y)) \\
& \leq \varphi^{-1}\left(\max _{\xi \in[s-h, s]} z(\xi, y)\right) \\
& (s, y) \in\left[\alpha_{*}\left(x_{0}\right), X\right) \times\left[y_{0}, Y\right) .
\end{aligned}
$$


It follows from (43), (44), and the definition of $z(x, y)$ that $z(x, y) \leq \widetilde{a}(X, Y)$

$$
\begin{gathered}
+\sum_{i=1}^{m} \int_{\alpha_{i}\left(x_{0}\right)}^{\alpha_{i}(x)} \int_{\beta_{i}\left(y_{0}\right)}^{\beta_{i}(y)} \tilde{f}_{i}(X, Y, s, t) \\
\times \tilde{\omega}_{i}\left(\varphi^{-1}(z(s, t))\right) d t d s \\
+\sum_{j=m+1}^{m+n} \int_{\alpha_{j}\left(x_{0}\right)}^{\alpha_{j}(x)} \int_{\beta_{i}\left(y_{0}\right)}^{\beta_{i}(y)} \tilde{f}_{i}(X, Y, s, t) \\
\times \tilde{\omega}_{j}\left(\varphi^{-1}\left(\max _{\xi \in[s-h, s]} z(\xi, t)\right)\right) d t d s, \\
(x, y) \in\left[x_{0}, X\right) \times\left[y_{0}, Y\right], \\
z(x, y) \leq \tilde{a}(X, Y), \quad(x, y) \in\left[\alpha_{*}\left(x_{0}\right)-h, x_{0}\right] \times\left[y_{0}, Y\right] .
\end{gathered}
$$

In order to demonstrate the basic condition of monotonicity, let $h(t):=\varphi^{-1}(t)$, which is clearly a continuous and nondecreasing function on $\mathbb{R}_{+}$. Thus, each $\widetilde{\omega}_{i}(h(t))$ is continuous and nondecreasing on $\mathbb{R}_{+}$and satisfies $\widetilde{\omega}_{i}(h(t))>0$ for $t>0$. Moreover, since $\widetilde{\omega}_{i}(t) \propto \widetilde{\omega}_{i+1}(t), \widetilde{\omega}_{i+1}(h(t)) / \widetilde{\omega}_{i}(h(t))$ is also continuous and nondecreasing on $\mathbb{R}_{+}$and positive on $(0,+\infty)$, implying that $\widetilde{\omega}_{i}(h(t)) \propto \widetilde{\omega}_{i+1}(h(t))$, for $i=$ $1,2, \ldots, m+n-1$. By Lemma 2 and (45),

$$
\begin{aligned}
z(x, y) \leq W_{m+n}^{-1} & \left(W\left(\widetilde{r}_{m+n}(X, Y, x, y)\right)\right. \\
& \left.+\int_{\alpha_{m+n}\left(x_{0}\right)}^{\alpha_{m+n}(x)} \int_{\beta_{m+n}\left(y_{0}\right)}^{\beta_{m+n}(y)} \tilde{f}_{m+n}(X, Y, s, t) d t d s\right),
\end{aligned}
$$

for $x_{0} \leq x<X_{2}$ and $y_{0} \leq y<Y_{2}$. It follows from (43) and (46) that

$$
\begin{aligned}
& u(x, y) \\
& \leq \varphi^{-1}\left(W _ { m + n } ^ { - 1 } \left(W\left(\widetilde{r}_{m+n}(X, Y, x, y)\right)\right.\right. \\
& \left.\left.+\int_{\alpha_{m+n}\left(x_{0}\right)}^{\alpha_{m+n}(x)} \int_{\beta_{m+n}\left(y_{0}\right)}^{\beta_{m+n}(y)} \tilde{f}_{m+n}(X, Y, s, t) d t d s\right)\right),
\end{aligned}
$$

for $x_{0} \leq x<X_{2}$ and $y_{0} \leq y<Y_{2}$. This proves the claimed (36).
Taking $x=X, y=Y, X_{2}=X_{1}$, and $Y_{2}=Y_{1}$ in (36), we have

$$
\begin{aligned}
& u(X, Y) \\
& \leq \varphi^{-1}\left(W _ { m + n } ^ { - 1 } \left(W_{m+n}\left(\widetilde{r}_{m+n}(X, Y, X, Y)\right)\right.\right. \\
& \left.\left.+\int_{\alpha_{m+n}\left(x_{0}\right)}^{\alpha_{m+n}(X)} \int_{\beta_{m+n}\left(y_{0}\right)}^{\beta_{m+n}(Y)} \tilde{f}_{m+n}(X, Y, s, t) d t d s\right)\right),
\end{aligned}
$$

for all $x_{0} \leq X<X_{1}, y_{0} \leq Y<Y_{1}$. It is easy to verify $\widetilde{r}_{m+n}(X, Y, X, Y)=r_{m+n}(X, Y)$. Thus, (48) can be written

$$
\begin{aligned}
& u(X, Y) \\
& \leq \varphi^{-1}\left(W _ { m + n } ^ { - 1 } \left(W_{m+n}\left(r_{m+n}(X, Y)\right)\right.\right. \\
& \left.\left.\quad+\int_{\alpha_{m+n}\left(x_{0}\right)}^{\alpha_{m+n}(X)} \int_{\beta_{m+n}\left(y_{0}\right)}^{\beta_{m+n}(Y)} \tilde{f}_{m+n}(X, Y, s, t) d t d s\right)\right) .
\end{aligned}
$$

Since $X, Y$ are arbitrary, replacing $X$ and $Y$ with $x$ and $y$, respectively, we get

$$
\begin{aligned}
& u(x, y) \\
& \leq \varphi^{-1}\left(W _ { m + n } ^ { - 1 } \left(W_{m+n}\left(r_{m+n}(x, y)\right)\right.\right. \\
& \left.\left.\quad+\int_{\alpha_{m+n}\left(x_{0}\right)}^{\alpha_{m+n}(x)} \int_{\beta_{m+n}\left(y_{0}\right)}^{\beta_{m+n}(y)} \tilde{f}_{m+n}(x, y, s, t) d t d s\right)\right),
\end{aligned}
$$

for all $(x, y) \in\left[x_{0}, X_{1}\right) \times\left[y_{0}, Y_{1}\right)$. This completes the proof.

\section{Applications}

In this section, we apply our result to prove the boundedness of solutions for a differential equation with the maxima.

Consider a system of partial differential equations with maxima

$$
\begin{array}{r}
\frac{\partial^{2} z(x, y)}{\partial x \partial y}=F\left(x, y, z(x, y), \max _{s \in[\alpha(x), \beta(x)]} \omega(z(s, y))\right), \\
(x, y) \in\left[x_{0}, x_{1}\right) \times\left[y_{0}, y_{1}\right) \\
z(x, y)=\psi(x, y), \quad(x, y) \in\left[\beta\left(x_{0}\right)-h, x_{0}\right] \times\left[y_{0}, y_{1}\right), \\
z\left(x, y_{0}\right)=f(x), \quad z\left(x_{0}, y\right)=g(y), \quad x \geq x_{0}, y \geq y_{0},
\end{array}
$$

where $F \in C\left(\left[x_{0}, x_{1}\right) \times\left[y_{0}, y_{1}\right) \times \mathbb{R}^{2}, \mathbb{R}\right), \omega \in C\left([0, \infty), \mathbb{R}_{+}\right)$, $\alpha, \beta \in C^{1}\left(\left[x_{0}, x_{1}\right), \mathbb{R}_{+}\right)$are nondecreasing such that $\alpha(x) \leq x$, 
$\beta(x) \leq x$, and $0<\beta(x)-\alpha(x) \leq h$ ( $h$ is a positive constant) for $x \in\left[x_{0}, x_{1}\right], \psi \in C\left(\left[\beta\left(x_{0}\right)-h, x_{0}\right] \times\left[y_{0}, y_{1}\right)\right.$, and $f \in$ $C\left(\left[x_{0}, x_{1}\right), \mathbb{R}\right), g \in C\left(\left[y_{0}, y_{1}\right), \mathbb{R}\right)$ satisfy that $f\left(x_{0}\right)=g\left(y_{0}\right)$ and $g(y)=\psi\left(x_{0}, y\right)$, for all $y \in\left[y_{0}, y_{1}\right)$.

Equation (51) is more general than the equation considered in Section 2.4 of [23]. The following result gives an estimate for its solutions.

Corollary 3. Suppose that functions $F$ and $\psi$ in (51) satisfy

$$
\begin{gathered}
|F(x, y, s, t)| \leq h_{1}(x, y) \mu_{1}(|s|)+h_{2}(x, y) \mu_{2}(|t|), \\
|\psi(x, y)| \leq|g(y)|, \quad \forall(x, y) \in\left[\beta\left(x_{0}\right)-h, x_{0}\right] \times\left[y_{0}, y_{1}\right),
\end{gathered}
$$

where $h_{i} \in C\left(\left[x_{0}, x_{1}\right) \times\left[y_{0}, y_{1}\right), \mathbb{R}_{+}\right)$and $\mu_{i} \in C\left(\mathbb{R}_{+},(0, \infty)\right)$, $i=1,2$. Then, any solution $z(x, y)$ of (51) has the estimate

$$
|z(x, y)| \leq Q_{2}^{-1}\left(Q_{2}(\gamma(x, y))+\int_{x_{0}}^{x} \int_{y_{0}}^{y} h_{2}(s, t) d t d s\right),
$$

for all $(x, y) \in\left[x_{0}, X_{1}\right) \times\left[y_{0}, Y_{1}\right)$, where

$$
\begin{gathered}
\gamma(x, y):=Q_{1}^{-1}\left(Q_{1}\left(\gamma_{1}(x, y)\right)+\int_{x_{0}}^{x} \int_{y_{0}}^{y} h_{1}(s, t) d t d s\right), \\
\gamma_{1}(x, y):=\max _{(\xi, \eta) \in\left[x_{0}, x\right] \times\left[y_{0}, y\right]}\left(\left|f(\xi)+g(\eta)-f\left(x_{0}\right)\right|\right), \\
Q_{2}(u):= \\
\int_{u_{2}}^{u} \frac{d s}{\left.\max _{\tau \in[0, s]}\left\{\tilde{\mu}_{2}(\widetilde{\omega}(\tau)) / \max _{\tau_{1} \in[0, \tau]}\left\{\mu_{1}\left(\tau_{1}\right)\right\}\right\} \max _{\tau \in[0, s]}\left\{\mu_{1}(\tau)\right\}\right\}}, \\
\quad Q_{1}(u):=\int_{u_{1}}^{u} \frac{d s}{\max _{\tau \in[0, s]}\left\{\mu_{1}(\tau)\right\}}, \\
\widetilde{\omega}(t):=\max _{s \in[0, t]}\{\omega(s)\}, \quad \widetilde{\mu}_{2}(t):=\max _{s \in[0, t]}\left\{\mu_{2}(s)\right\} \text {, and }
\end{gathered}
$$

$X_{1}, Y_{1}$ are given as in Theorem 1 , and constants $u_{1}>0, u_{2}>0$ are given arbitrarily.

Proof. From (51), we obtain

$$
\begin{aligned}
& z(x, y) \\
& =f(x)+g(y)-f\left(x_{0}\right) \\
& +\int_{x_{0}}^{x} \int_{y_{0}}^{y} F\left(s, t, z(s, t), \max _{\xi \in[\alpha(s), \beta(s)]} \omega(z(\xi, t))\right) d t d s, \\
& (x, y) \in \Lambda \text {, } \\
& z(x, y)=\psi(x, y), \\
& (x, y) \in(x, y) \in\left[\beta\left(x_{0}\right)-h, x_{0}\right] \times\left[y_{0}, y_{1}\right) .
\end{aligned}
$$

From (52) and (55), we get

$$
\begin{aligned}
& |z(x, y)| \\
& \leq\left|f(x)+g(y)-f\left(x_{0}\right)\right| \\
& \quad+\int_{x_{0}}^{x} \int_{y_{0}}^{y} h_{1}(s, t) \mu_{1}(|z(s, t)|) d t d s \\
& \quad+\int_{x_{0}}^{x} \int_{y_{0}}^{y} \mu_{2}\left(\left|\max _{\xi \in[\alpha(s), \beta(s)]} \omega(z(\xi, t))\right|\right) d t d s \\
& \leq\left|f(x)+g(y)-f\left(x_{0}\right)\right| \\
& \quad+\int_{x_{0}}^{x} \int_{y_{0}}^{y} h_{1}(s, t) \mu_{1}(|z(s, t)|) d t d s \\
& \quad+\int_{x_{0}}^{x} \int_{y_{0}}^{y} h_{2}(s, t) \widetilde{\mu}_{2}\left(\max _{\xi \in[\alpha(s), \beta(s)]} \widetilde{\omega}(|z(\xi, t)|) d t d s,\right. \\
& |z(x, y)| \leq|\psi(x, y)|, \\
& (x, y) \in\left[\beta\left(x_{0}\right)-h, x_{0}\right] \times\left[y_{0}, y_{1}\right) .
\end{aligned}
$$

Set $v(x, y)=|z(x, y)|$ for $(x, y) \in\left[\beta\left(x_{0}\right)-h, x_{1}\right) \times\left[y_{0}, y_{1}\right)$. Noting that $\max _{\xi \in[\alpha(s), \beta(s)]} z(\xi, y) \leq \max _{\xi \in[\beta(s)-h, \beta(s)]} z(\xi, y)$, from (56), we get

$$
\begin{gathered}
v(x, y) \leq \gamma_{1}(x, y)+\int_{x_{0}}^{x} \int_{y_{0}}^{y} h_{1}(s, t) \mu_{1}(v(s, t)) d t d s \\
+\int_{\beta\left(x_{0}\right)}^{\beta(x)} \int_{y_{0}}^{y} h_{2}\left(\beta^{-1}(\eta), t\right)\left(\beta^{-1}(\eta)\right)^{\prime} \tilde{\mu}_{2} \\
\times\left(\max _{\xi \in[\eta-h, \eta]} \widetilde{\omega}(v(\xi, t)) d t d \eta\right. \\
v(x, y) \leq|\psi(x, y)|, \quad(x, y) \in\left[\beta\left(x_{0}\right)-h, x_{0}\right] \times\left[y_{0}, y_{1}\right) .
\end{gathered}
$$

Applying Theorem 1 to specified $m=n=1, \varphi(u)=u$, $f_{1}(x, y, s, t)=h_{1}(s, t), \alpha_{1}(t)=t$, and $\alpha_{2}(t)=\beta(t), \beta_{i}(t)=$ $t, i=1,2, f_{2}(s, t)=h_{2}\left(\beta^{-1}(s), t\right)\left(\beta^{-1}(s)\right)^{\prime}$, and $\omega_{i}(u)=$ $\mu_{i}(u), i=1,2$, we obtain (53) from (57). (51).

Next, we discuss the uniqueness of solutions for system

Corollary 4. Suppose that $g(s)=s$ and

$$
\begin{aligned}
& \left|F\left(x, y, s_{1}, t_{1}\right)-F\left(x, y, s_{2}, t_{2}\right)\right| \\
& \quad \leq h_{1}(x, y) \mu_{1}\left(\left|x_{1}-x_{2}\right|\right)+h_{2}(x, y) \mu_{2}\left(\left|y_{1}-y_{2}\right|\right),
\end{aligned}
$$

for all $(x, y) \in\left[x_{0}, x_{1}\right] \times\left[y_{0}, y_{1}\right]$ and all $x_{i}, y_{i} \in \mathbb{R}(i=1,2)$, where $h_{i} \in C\left(\left[x_{0}, x_{1}\right] \times\left[y_{0}, y_{1}\right], \mathbb{R}_{+}\right)$and $\mu_{i} \in C\left(\left[\mathbb{R}, \mathbb{R}_{+}\right)\right.$are 
both nondecreasing such that $\mu_{i}(0)=0, \mu_{i}(u)>0$ for $u>0$, $\mu_{2} / \mu_{1}$ is also nondecreasing, and $\int_{0}^{1} d s / \mu_{i}(s)=+\infty, i=1,2$. Then, system (51) has at most one solution on $\left[x_{0}, x_{1}\right) \times\left[y_{0}, y_{1}\right)$.

Proof. $g(s)=s$. From (51), we get

$$
\begin{gathered}
\frac{\partial^{2} z(x, y)}{\partial x \partial y}=F\left(x, y, z(x, y), \max _{s \in[\alpha(x), \beta(x)]} z(s, y)\right), \\
(x, y) \in\left[x_{0}, x_{1}\right) \times\left[y_{0}, y_{1}\right), \\
z(x, y)=\psi(x, y), \quad(x, y) \in\left[\alpha\left(x_{0}\right)-h, x_{0}\right] \times\left[y_{0}, y_{1}\right] \\
z\left(x, y_{0}\right)=f(x), z\left(x_{0}, y\right)=g(y), \quad x \geq x_{0}, y \geq y_{0} .
\end{gathered}
$$

Assume that (59) has two different solutions $u(x, y)$ and $v(x, y)$. From the equivalent integral equation system (55), we have

$$
\begin{aligned}
& |u(x, y)-v(x, y)| \\
& \leq \int_{x_{0}}^{x} \int_{y_{0}}^{y} h_{1}(s, t) \mu_{1}(|u(s, t)-v(s, t)|) d t d s \\
& \quad+\int_{x_{0}}^{x} \int_{y_{0}}^{y} h_{2}(s, t) \mu_{2}\left(\max _{\xi \in[\alpha(s), \beta(s)]} u(\xi, t)\right. \\
& \leq \int_{x_{0}}^{x} \int_{y_{0}}^{y} h_{1}(s, t) \mu_{1}(|u(s)-v(s)|) d t d s \\
& \quad+\int_{x_{0}}^{x} \int_{y_{0}}^{y} h_{2}(s, t) \mu_{2} \\
& \times\left(\max _{\xi \in[\alpha(s), \beta(s)]}|u(\xi, t)|\right) d t d s
\end{aligned}
$$

for all $(x, y) \in\left[x_{0}, x_{1}\right] \times\left[y_{0}, y_{1}\right]$. The continuity of the function $u(x, y)$ implies that for any fixed points $s \in\left[x_{0}, x\right]$ and $t \in\left[y_{0}, y\right]$ there exists a point $\eta \in[\alpha(s), \beta(s)]$ such that the inequality $\max _{\xi \in[\alpha(s), \beta(s)]} u(\xi, t)=u(\eta, t)$ holds, and therefore

$$
\begin{aligned}
& \left|\max _{\xi \in[\alpha(s), \beta(s)]} u(\xi, t)-\max _{\xi \in[\alpha(s), \beta(s)]} v(\xi, t)\right| \\
& \quad=\left|u(\eta, t)-\max _{\xi \in[\alpha(s), \beta(s)]} v(\xi, t)\right| \\
& \quad \leq|u(\eta, t)-v(\eta, t)| \leq \max _{\xi \in[\alpha(s), \beta(s)]}|u(\xi, t)-v(\xi, t)| .
\end{aligned}
$$

Hence,

$$
\begin{aligned}
& |u(x, y)-v(x, y)| \\
& \quad \leq \int_{x_{0}}^{x} \int_{y_{0}}^{y} h_{1}(s, t) \mu_{1}(|u(s, y)-v(s, y)|) d t d s \\
& \quad+\int_{x_{0}}^{x} \int_{y_{0}}^{y} h_{2}(s, t) \mu_{2}\left(\max _{\xi \in[\alpha(s), \beta(s)]}|u(\xi, t)-v(\xi, t)|\right) d s .
\end{aligned}
$$

Let

$$
\begin{array}{r}
\phi(x, y):=|u(x, y)-v(x, y)|, \\
(x, y) \in\left[\beta\left(x_{0}\right)-h, x_{1}\right] \times\left[y_{0}, y_{1}\right] .
\end{array}
$$

Because $\max _{\xi \in[\alpha(s), \beta(s)]} u(\xi, y) \leq \max _{\xi \in[\beta(s)-h, \beta(s)]} u(\xi, y)$, from (62), we obtain

$$
\begin{aligned}
& \phi(x, y) \leq \int_{x_{0}}^{x} \int_{y_{0}}^{y} h_{1}(s, t) \mu_{1}(\phi(s, t)) d t d s \\
&+\int_{\beta\left(x_{0}\right)}^{\beta(x)} \int_{y_{0}}^{y} h_{2}\left(\beta^{-1}(\eta), t\right)\left(\beta^{-1}(\eta)\right)^{\prime} \\
& \times \mu_{2}\left(\max _{\xi \in[\eta-h, \eta]} \phi(\xi, t)\right) d t d \eta, \\
&(x, y) \in\left[x_{0}, x_{1}\right) \times\left[y_{0}, y_{1}\right), \\
& \phi(x, y) \leq 0, \quad(x, y) \in\left[\beta\left(x_{0}\right)-h, x_{0}\right] \times\left[y_{0}, y_{1}\right) .
\end{aligned}
$$

Applying Theorem 1 to specified $m=n=1, \varphi(u)=u$, $f_{1}(s, t)=h_{1}(s, t), \alpha_{1}(t)=t, \alpha_{2}(t)=\beta(t), f_{2}(s, t)=$ $h_{2}\left(\beta^{-1}(t), s\right)\left(\beta^{-1}(t)\right)^{\prime}, g(t)=t, a(x, y)=0$, and $\omega_{i}(t)=$ $\mu_{\mathrm{i}}(t), i=1,2$, from (64), we obtain

$$
\phi(x, y) \leq \widehat{Q}_{2}^{-1}\left(\widehat{Q}_{2}\left(\bar{\gamma}_{2}(s, t)\right)+\int_{x_{0}}^{x} \int_{y_{0}}^{y} h_{2}(s, t) d t d s\right),
$$

for all $(x, y) \in\left[x_{0}, X_{1}\right] \times\left[y_{0}, Y_{1}\right]$, where

$$
\begin{gathered}
\widehat{Q}_{1}(u):=\int_{1}^{u} \frac{d s}{\mu_{1}(s)}, \quad \widehat{Q}_{2}(u):=\int_{1}^{u} \frac{d s}{\mu_{2}(\tau)}, \\
\bar{r}_{1}(x, y):=0 \\
\bar{r}_{2}(x, y):=\widehat{Q}_{1}^{-1}\left(\widehat{Q}_{1}\left(\bar{r}_{1}(x, y)\right)+\int_{x_{0}}^{x} \int_{y_{0}}^{y} h_{1}(s, t) d t d s\right) .
\end{gathered}
$$

By the definition of $\widehat{Q}_{i}$ and properties of $\mu_{i}$, noting that $\int_{0}^{1} d s / \mu_{i}(s)=+\infty(i=1,2)$, we obtain

$$
\lim _{u \rightarrow 0^{+}} \widehat{Q}_{i}(u)=-\infty, \quad \lim _{u \rightarrow-\infty} \widehat{Q}_{i}^{-1}(u)=0, \quad i=1,2 .
$$

Since $\int_{x_{0}}^{x} \int_{y_{0}}^{y} h_{1}(s, t) d t d s$ is finite on a finite interval, $\left[x_{0}, x_{1}\right]$ and $\left[y_{0}, y_{1}\right]$, by $(67)$, we obtain

$$
\widehat{Q}_{1}\left(\bar{r}_{1}(x, y)\right)+\int_{x_{0}}^{x} \int_{y_{0}}^{y} h_{1}(s, t) d t d s=-\infty .
$$


Thus, we obtain $\bar{\gamma}_{2}(x, y)=0$ from (68), (69), and (70) immediately. Similarly, noting that $\int_{x_{0}}^{x} \int_{y_{0}}^{y} h_{2}(s, t) d t d s$ is finite on finite interval, $\left[x_{0}, x_{1}\right]$ and $\left[y_{0}, y_{1}\right]$, from (69), we obtain

$$
\widehat{Q}_{2}\left(\bar{r}_{2}(x, y)\right)+\int_{x_{0}}^{x} \int_{y_{0}}^{y} h_{2}(s, t) d t d s=-\infty .
$$

Thus, we conclude from (65), (69), and (71) that $\mid u(x, y)-$ $v(x, y) \mid \leq 0$, which implies that $u(x, y)=v(x, y)$, for all $(x, y) \in\left[x_{0}, X_{1}\right) \times\left[y_{0}, Y_{1}\right)$, where $X_{1}, Y_{1}$ are given as in Theorem 1 . The uniqueness is proved.

\section{Acknowledgments}

The author is grateful to the reviewer for his valuable suggestions. This research was supported by Scientific Research Fund of Sichuan Provincial Education Department of China.

\section{References}

[1] T. H. Gronwall, "Note on the derivatives with respect to a parameter of the solutions of a system of differential equations," Annals of Mathematics, vol. 20, no. 4, pp. 292-296, 1919.

[2] R. Bellman, "The stability of solutions of linear differential equations," Duke Mathematical Journal, vol. 10, pp. 643-647, 1943.

[3] I. A. Bihari, "A generalization of a lemma of Bellman and its application to uniqueness problems of differential equations," Acta Mathematica Academiae Scientiarum Hungaricae, vol. 7, pp. 81-94, 1956.

[4] O. Lipovan, "A retarded Gronwall-like inequality and its applications," Journal of Mathematical Analysis and Applications, vol. 252, no. 1, pp. 389-401, 2000.

[5] R. P. Agarwal, S. Deng, and W. Zhang, "Generalization of a retarded Gronwall-like inequality and its applications," Applied Mathematics and Computation, vol. 165, no. 3, pp. 599-612, 2005.

[6] M. Pinto, "Integral inequalities of Bihari-type and applications," Funkcialaj Ekvacioj, vol. 33, no. 3, pp. 387-403, 1990.

[7] W.-S. Wang, "A generalized retarded Gronwall-like inequality in two variables and applications to BVP," Applied Mathematics and Computation, vol. 191, no. 1, pp. 144-154, 2007.

[8] D. D. Baĭnov and P. S. Simeonov, Integral Inequalities and Applications, Kluwer Academic, Dodrecht, The Netherlands, 1992.

[9] B. G. Pachpatte, "Explicit bounds on certain integral inequalities," Journal of Mathematical Analysis and Applications, vol. 267, no. 1, pp. 48-61, 2002.

[10] Y.-H. Kim, "Gronwall, Bellman and Pachpatte type integral inequalities with applications," Nonlinear Analysis: Theory, Methods \& Applications, vol. 71, no. 12, pp. e2641-e2656, 2009.

[11] W.-S. Cheung and Q.-H. Ma, "On certain new GronwallOu-Iang type integral inequalities in two variables and their applications," Journal of Inequalities and Applications, vol. 2005, Article ID 541438, 2005.

[12] O. Lipovan, "A retarded integral inequality and its applications," Journal of Mathematical Analysis and Applications, vol. 285, no. 2, pp. 436-443, 2003.

[13] Q.-H. Ma and E.-H. Yang, "On some new nonlinear delay integral inequalities," Journal of Mathematical Analysis and Applications, vol. 252, no. 2, pp. 864-878, 2000.
[14] B. G. Pachpatte, "On some new inequalities related to certain inequalities in the theory of differential equations," Journal of Mathematical Analysis and Applications, vol. 189, no. 1, pp. 128144, 1995.

[15] B. G. Pachpatte, Inequalities for Differential and Integral Equations, Academic Press, London, UK, 1998.

[16] B. G. Pachpatte, Integral and Finite Difference Inequalities and Applications, vol. 205 of North-Holland Mathematics Studies, Elsevier, Amsterdam, The Netherlands, 2006.

[17] W. Zhang and S. Deng, "Projected Gronwall-Bellman's inequality for integrable functions," Mathematical and Computer Modelling, vol. 34, no. 3-4, pp. 393-402, 2001.

[18] L. Horváth, "Generalized Bihari type integral inequalities and the corresponding integral equations," Journal of Inequalities and Applications, vol. 2009, Article ID 409809, 20 pages, 2009.

[19] L. Ou-Iang, "The boundedness of solutions of linear differential equations $y^{\prime \prime}+A(t) y^{\prime}=0$," Advances in Mathematics (China), vol. 3, pp. 409-418, 1957.

[20] V. G. Angelov and D. D. Bainnov, "On the functional-differential equations with 'maximums"' Applicable Analysis, vol. 16, no. 3, pp. 187-194, 1983.

[21] A. Golev, S. Hristova, and A. Rahnev, "An algorithm for approximate solving of differential equations with 'maxima", Computers \& Mathematics with Applications, vol. 60, no. 10, pp. 2771-2778, 2010.

[22] E. P. Popov, Automatic Regulation and Control, Nauka, Moscow, Russia, 1966.

[23] D. Bainov and S. Hristova, Differential Equations Equations with Maxima, CRC \& Taylor \& Francis, Boca Raton, Fla, USA, 2011.

[24] D. Bainov and E. Minchev, "Forced oscillations of solutions of hyperbolic equations of neutral type with maxima," Applicable Analysis, vol. 70, no. 3-4, pp. 259-267, 1999.

[25] D. P. Mishev and S. M. Musa, "Distribution of the zeros of the solutions of hyperbolic differential equations with maxima," The Rocky Mountain Journal of Mathematics, vol. 37, no. 4, pp. 12711281, 2007.

[26] M. Bohner, S. Hristova, and K. Stefanova, "Nonlinear integral inequalities involving maxima of the unknown scalar functions," Mathematical Inequalities and Applications, vol. 15, no. 4, pp. 811-825, 2012.

[27] J. Henderson and S. Hristova, "Nonlinear integral inequalities involving maxima of unknown scalar functions," Mathematical and Computer Modelling, vol. 53, no. 5-6, pp. 871-882, 2011.

[28] S. G. Hristova and K. V. Stefanova, "Linear integral inequalities involving maxima of the unknown scalar functions," Journal of Mathematical Inequalities, vol. 4, no. 4, pp. 523-535, 2010.

[29] S. G. Hristova and K. V. Stefanova, "Some integral inequalities with maximum of the unknown functions," Advances in Dynamical Systems and Applications, vol. 6, no. 1, pp. 57-69, 2011. 


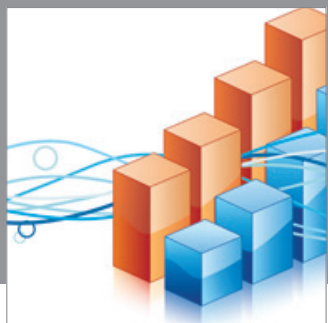

Advances in

Operations Research

mansans

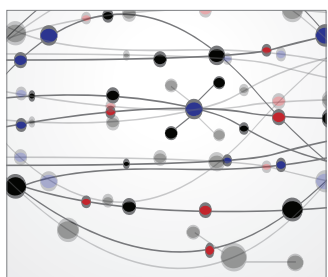

The Scientific World Journal
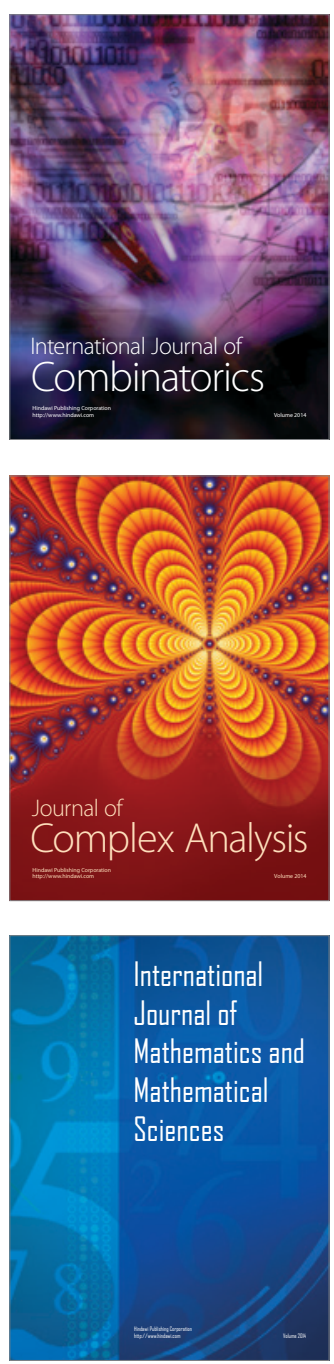
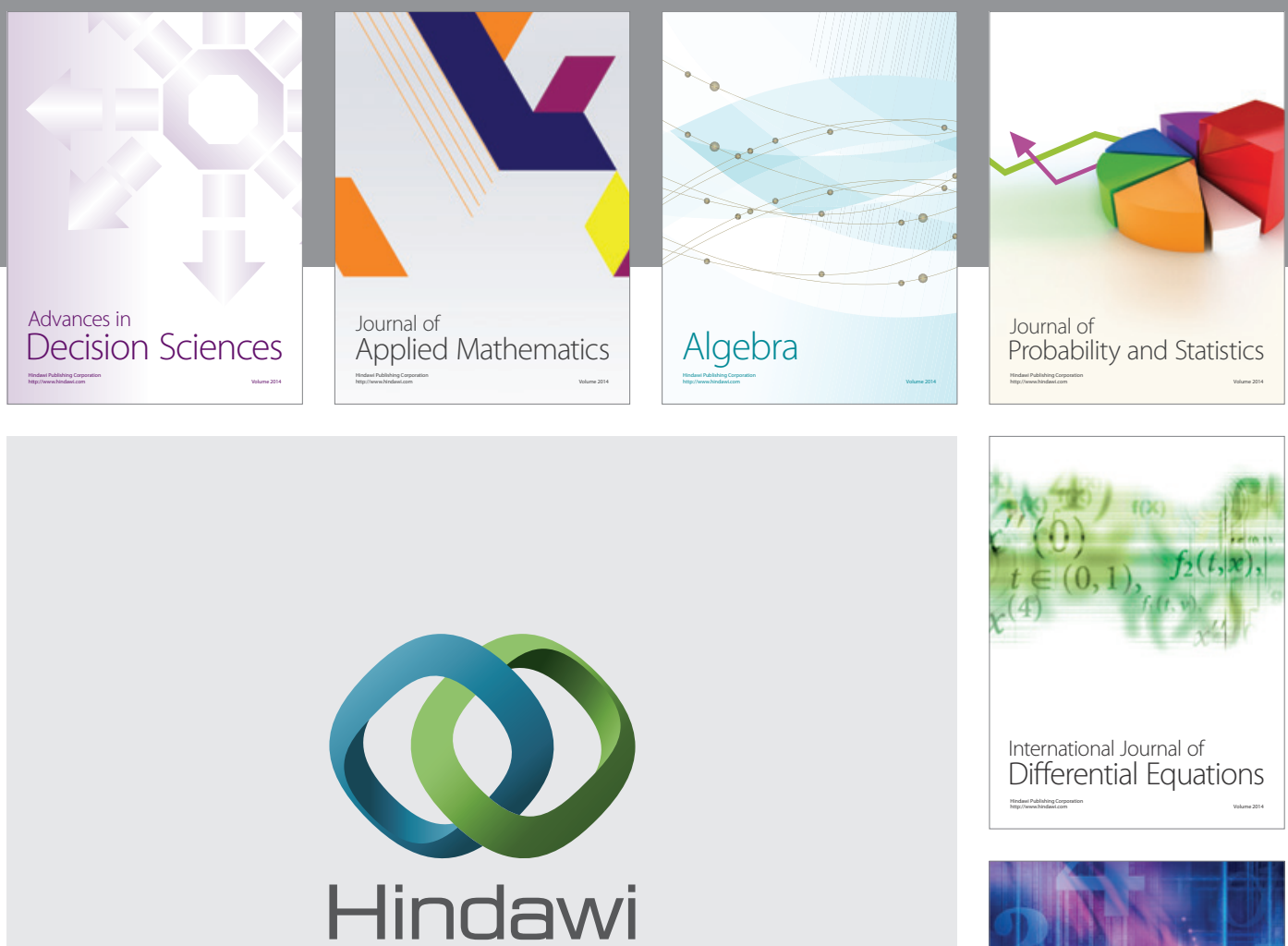

Submit your manuscripts at http://www.hindawi.com
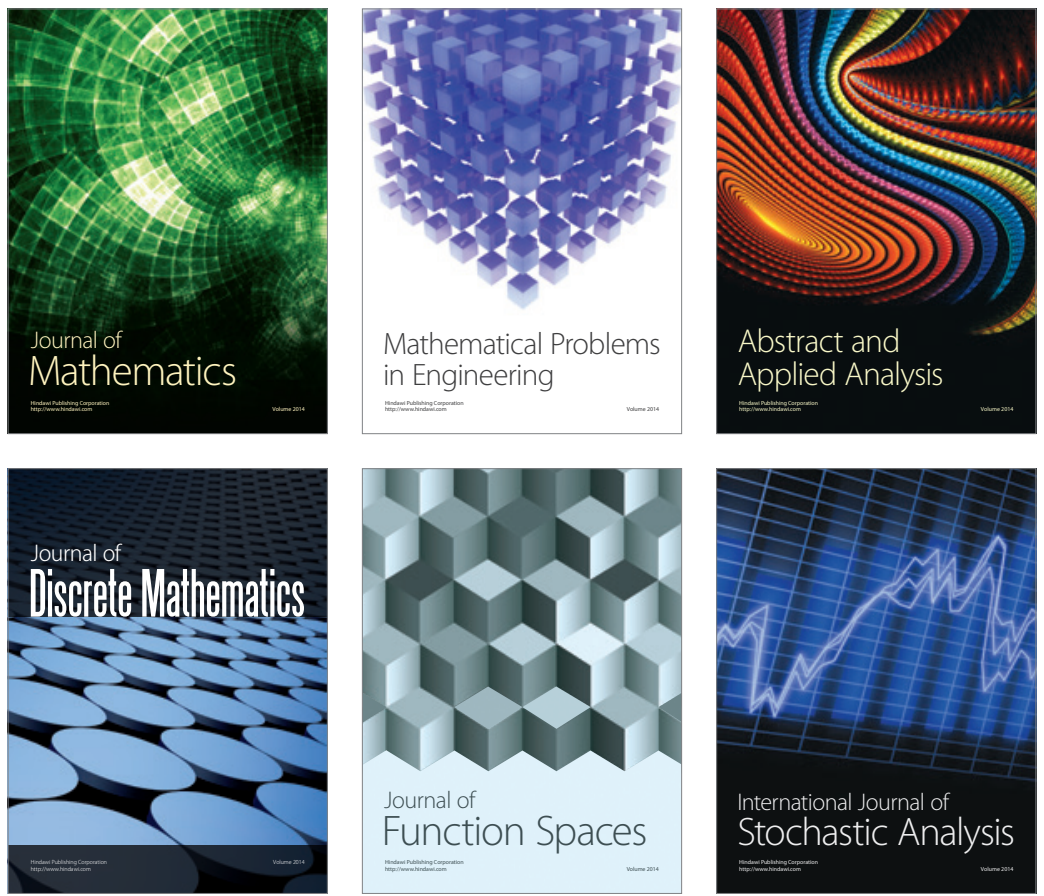

Journal of

Function Spaces

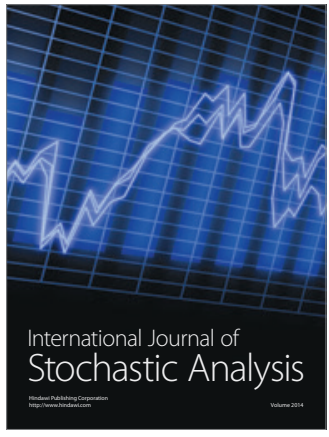

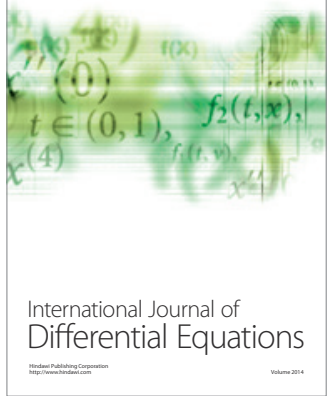
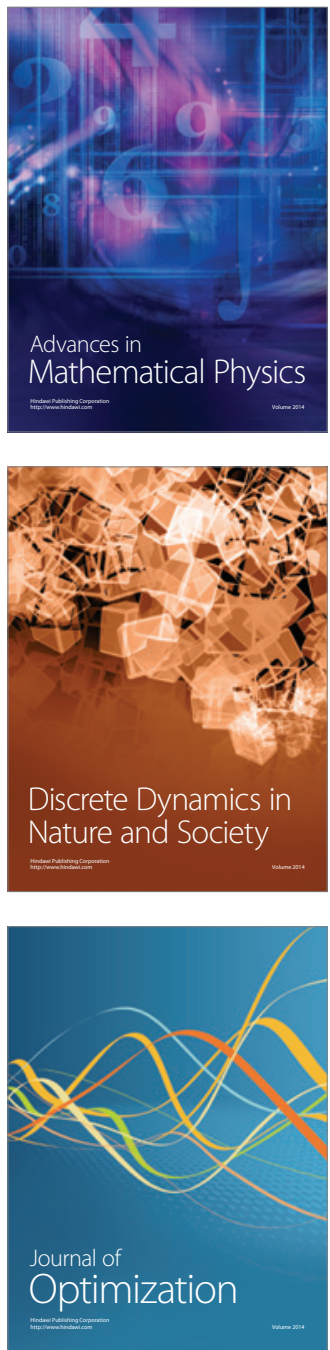